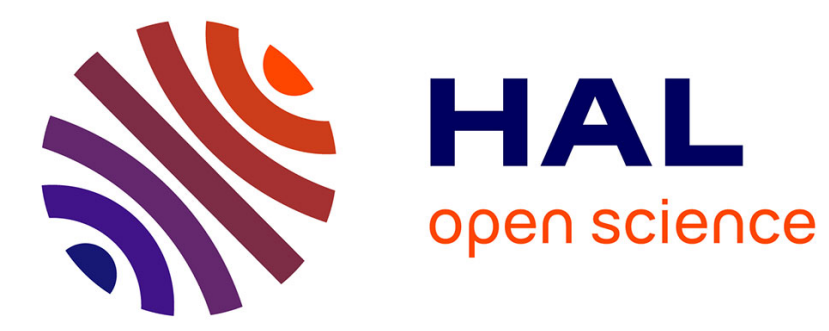

\title{
Dielectric scaling near a percolation threshold
}

Ludovic Benguigui

\section{To cite this version:}

Ludovic Benguigui. Dielectric scaling near a percolation threshold. Journal de Physique Lettres, 1985, 46 (21), pp.1015-1021. 10.1051/jphyslet:0198500460210101500 . jpa-00232929

\section{HAL Id: jpa-00232929 https://hal.science/jpa-00232929}

Submitted on 1 Jan 1985

HAL is a multi-disciplinary open access archive for the deposit and dissemination of scientific research documents, whether they are published or not. The documents may come from teaching and research institutions in France or abroad, or from public or private research centers.
L'archive ouverte pluridisciplinaire HAL, est destinée au dépôt et à la diffusion de documents scientifiques de niveau recherche, publiés ou non, émanant des établissements d'enseignement et de recherche français ou étrangers, des laboratoires publics ou privés. 
Classification

Physics Abstracts

$77.40-64.60$

\section{Dielectric scaling near a percolation threshold}

\section{Benguigui}

Solid State Institute and Department of Physics, Technion, Israel Institute of Technology, Haifa, 32000, Israel

(Reçu le 24 juillet 1985, accepté le 17 septembre 1985)

Résumé. - On a mesuré la constante diélectrique complexe d'un système métal-isolant percolant, au-dessous de $p_{\mathrm{c}}$, à deux et trois dimensions. A 2D on observe un accord parfait avec la théorie des lois d'échelle, mais à 3D, l'accord n'est bon qu'à basse fréquence.

Abstract. - We measured the complex dielectric constant of a percolative metal-insulator system below $p_{c}$, in two and three dimensions. In 2D, a perfect agreement with the scaling theory is observed, but in $3 \mathrm{D}$ the agreement is good only at low frequency.

The AC conduction of a metal-insulator system near the percolation threshold is an important problem, but it has received attention essentially from theoreticians [1-3]. The motivation of the present work comes from this situation. We present in this Letter dielectric measurements in a relatively large interval of frequencies $\left(10^{2}-10^{7} \mathrm{~Hz}\right)$ on a percolative system in $2 \mathrm{D}$ and 3D. We compared the results with the scaling approach and found that there is an excellent agreement except in 3D at high frequency. It is not clear what is the origin of this discrepancy.

In the scaling approach [2], a scaling function for $\varepsilon$ (the complex dielectric constant of the metal-insulator system) has been proposed

$$
\frac{\varepsilon \omega}{i \sigma_{0}}=\left|p-p_{\mathrm{c}}\right|^{t} F\left(\frac{\frac{\varepsilon_{0} \omega}{i \sigma_{0}}}{\left|p-p_{\mathrm{c}}\right|^{t+s}}\right) .
$$

In (1) $\varepsilon_{0}$ is the dielectric constant of the insulator, $\sigma_{0}$, the conductivity of the metal and $p$ is the fraction of the metal. $t$ and $s$ are the well known conductivity exponent $\left(p>p_{\mathrm{c}}\right)$ and dielectric exponent $\left(p<p_{\mathrm{c}}\right)$. The scaling function $F$ has the following asymptotic forms. If $\omega \varepsilon_{0} / \sigma_{0} \ll$ $\left|p-p_{\mathrm{c}}\right|^{t+s}$ (the low frequency limit) $F$ is given by

$$
\begin{array}{ll}
p>p_{\mathrm{c}} & F(z)=A+B z+C z^{2} \\
p<p_{\mathrm{c}} & F(z)=B^{\prime} z+C^{\prime} z^{2} .
\end{array}
$$

If now $\omega \varepsilon_{0} / \sigma_{2} \gg\left|p-p_{\mathrm{c}}\right|^{t+s}$, it was proposed that

$$
F(z)=A^{\prime \prime} z^{\frac{t}{t+s}}
$$


From these forms of $F$, it is possible to see that in the limit $\omega \rightarrow 0, \varepsilon_{1}$ (the real part of $\varepsilon$ ) diverges for $p \rightarrow p_{\mathrm{c}}$ with the exponent $s$, above and below $p_{\mathrm{c}}$ and that in the high frequency limit

$$
p \gtrless p_{c} \quad \varepsilon_{1} \sim \varepsilon_{2} \sim \omega^{-s / t+s}
$$

( $\varepsilon_{2}$ is the imaginary part of $\varepsilon$ ), independent of $p$. It is clear that (4) can be valid only in some interval of frequency, since for $\omega$ very large $\varepsilon_{1}$ goes to a constant value. This defines a frequency limit $\omega_{\infty}$ for the validity of (4). Between the two limits $\omega \ll \omega_{p}\left(\omega_{p}=\sigma_{0} / \varepsilon_{0}\left|p-p_{\mathrm{c}}\right|^{t+s}\right)$ and $\omega \gg \omega_{p}$ exists an interval of frequency, which encloses the relaxation frequency $\omega_{\mathrm{R}}$ of the system. Below $p_{c}$, the free charges of the metal relax in order to make the field null in the metal. $\omega_{R}$ is also the frequency maximum of $\varepsilon_{2}(\omega)$. Since at $p_{c}, \varepsilon_{2}(\omega)$ diverges for $\omega \rightarrow 0$, it is clear that $\omega_{R}$ goes to zero for $p \rightarrow p_{\mathrm{c}}$. In the scaling theory there is only one time scale $\left(\tau \sim \omega_{p}^{-1}\right)$ and it is expected that $\omega_{\mathrm{R}}$ goes to zero like $\left|p-p_{\mathrm{c}}\right|^{t+s}$.

The experimental system is made of balls of glass and iron, with a diameter of $1.2-1.5 \mathrm{~mm}$. For the size of the sample and their preparation, we used the results of Powell [4] and Ottavi et al. [5]. The distance between the electrodes and their size are taken at least 30 times the diameter of the balls. In $3 \mathrm{D}$ the measuring cell is a brass cylinder, which can contain $10^{5}$ balls approximately. In 2D, we took 8 identical samples, mounted in parallel. Each of them is made of two concentric electrodes with a layer of mixture (height of 1.5 time the ball diameter) between them. Each layer is supported by a sheet of plexiglass.

We performed measurements from $230 \mathrm{~Hz}$ to $2 \times 10^{7} \mathrm{~Hz}$ by means of an automatic bridge (General Radio LC 1688) and from $5 \times 10^{4}$ to $10^{7} \mathrm{~Hz}$ using a Q meter of Boonton. Before the measurements, the balls were dried to eliminate the humidity, which gives a spurious conductivity. All the results presented in this Letter are taken below $p_{c}$. Above $p_{c}$, the measurements are difficult, because the large conductivity and also because we observed instabilities. Some results in 3D have already been published [6].

In figures 1 to 4 , we show the variations of $\varepsilon_{1}$ and $\varepsilon_{2}$ as functions of $\omega$ for some values of $p$, in $2 \mathrm{D}$ and in $3 \mathrm{D}$. For the $2 \mathrm{D}$ measurements, $\varepsilon_{2}$ was determined by two methods. First, directly from the measurement of the sample impedance, but the results are scattered. Since the scatter in $\varepsilon_{1}$ is not very important, we calculated $\varepsilon_{2}(\omega)$ from $\varepsilon_{1}(\omega)$ through the Kramer-Kronig relation. At the same time, we made automatically the correction of $\varepsilon_{2}$ from the conductivity of the glass.

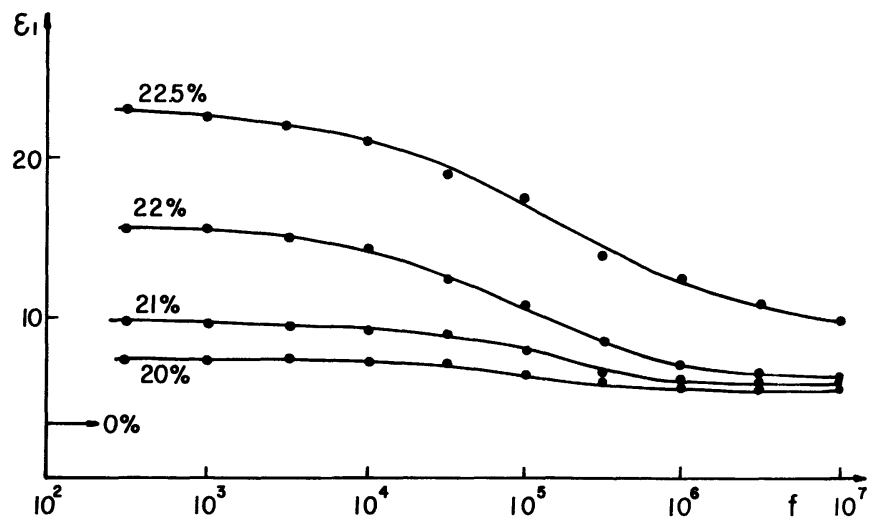

Fig. 1. $-\varepsilon_{1}$ versus the frequency for 3D samples. 


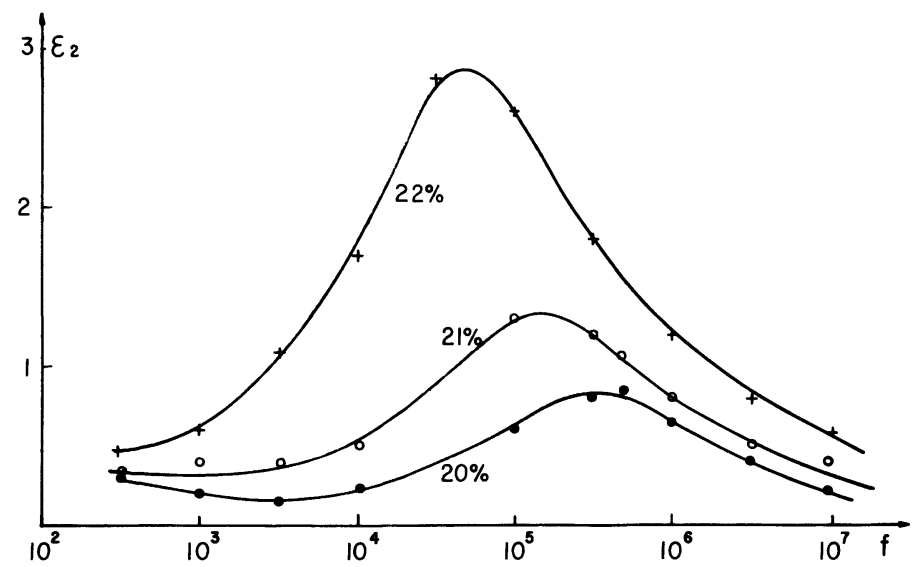

Fig. 2. $-\varepsilon_{2}$ versus the frequency for $3 \mathrm{D}$ samples.

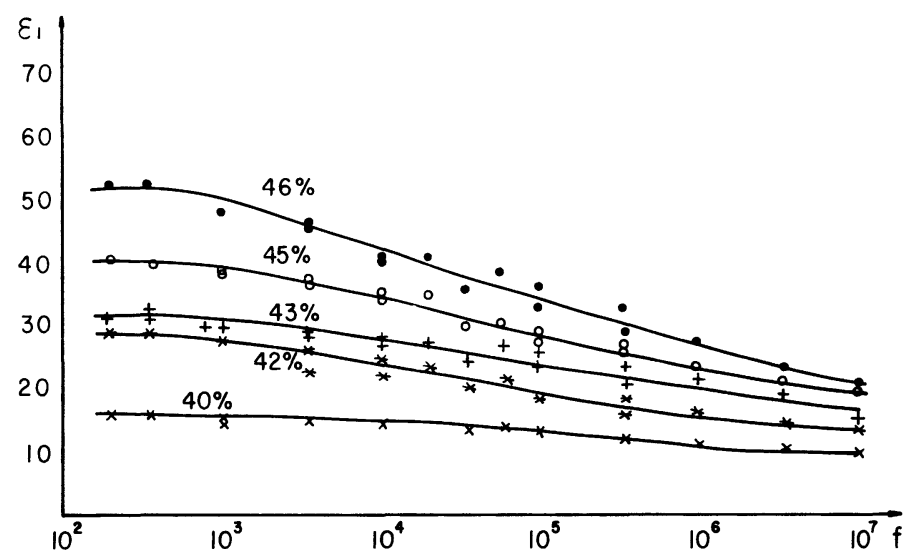

Fig. 3. $-\varepsilon_{1}$ versus the frequency for $2 \mathrm{D}$ samples.

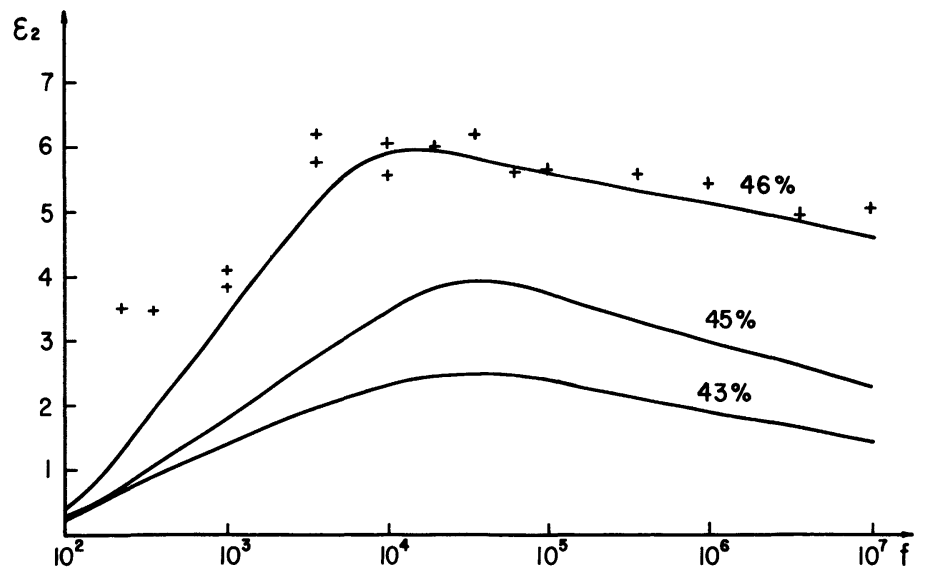

Fig. 4. $-\varepsilon_{2}$ versus the frequency for $2 \mathrm{D}$ samples. The lines are calculated from the values of $\varepsilon_{1}(\omega)$ using the Kramer-Kronig relation. The points are from the direct measurement of $\varepsilon_{2}$. Remark the large scatter of the points. At low frequency the points are not near the line, because of the residual resistivity of the glass. 
The threshold $p_{\mathrm{c}}$ was determined by fitting the values (shown in Fig. 5) of $\varepsilon_{1}(\omega \rightarrow 0)$ with the expression $\varepsilon_{1}(\omega \rightarrow 0) \sim\left|p-p_{\mathrm{c}}\right|^{-s}$. In 3D, we find $s=0.76 \pm 0.02$ and $p_{\mathrm{c}}=0.235$ and in $2 \mathrm{D}, s=1.35 \pm 0.05$ and $p_{\mathrm{c}}=0.5$. The values of $s$ are in excellent agreement with other determination of this exponent.

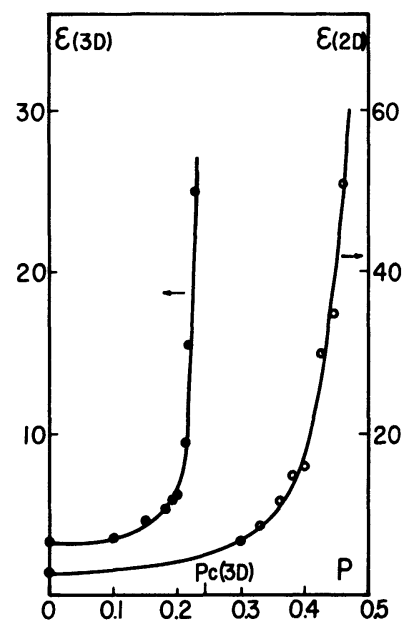

Fig. 5. - Divergence of the low frequency limit of $\varepsilon_{1}$.

In figure 6, we drew the variation of $\omega_{\mathrm{R}}$ as function of $\left|p-p_{\mathrm{c}}\right|$ and we determined the exponent $R, \omega_{\mathrm{R}} \simeq\left|p-p_{\mathrm{c}}\right|^{R}$. We got $R(3 \mathrm{D})=2 \pm 0.2$ and $R(2 \mathrm{D})=2.3 \pm 0.5$. If the value of $R(2 \mathrm{D})$ is consistent with $t+s=2.7$, in $3 \mathrm{D}$ it seems that $R$ is different from $t+s \simeq 2.6$ - if one takes $t \sim 1.9$.

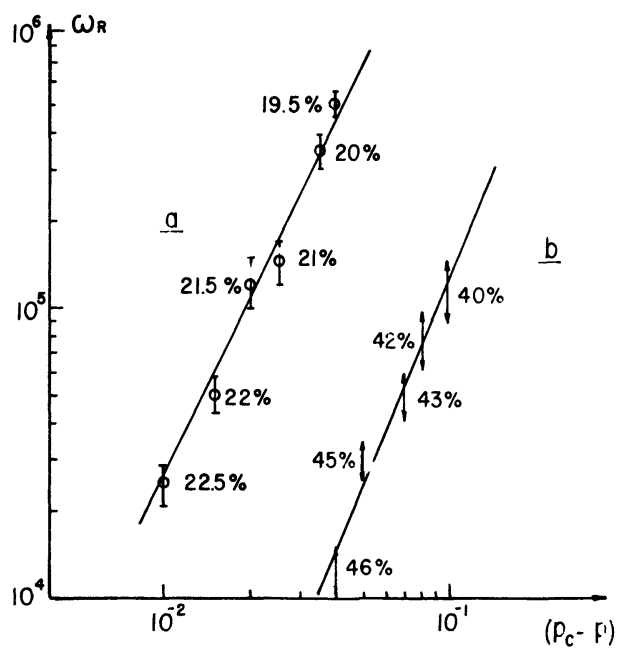

Fig. 6. - Relaxation frequency versus $\left|p_{c}-p\right|$; a) in 3D, b) in 2D. 
In order to compare our results with scaling we proceeded in the following way. From (1) it is easy to see that $\varepsilon_{1}$ and $\varepsilon_{2}$ can be expressed as

$$
(k=1,2) \quad \frac{\varepsilon_{k}}{\varepsilon_{0}}=\frac{\left|p-p_{\mathrm{c}}\right|^{t}}{\omega \tau_{0}} B_{k}\left(\frac{\omega \tau_{0}}{\left|p-p_{\mathrm{c}}\right|^{t+s}}\right)
$$

where $\tau_{0}=\varepsilon_{0} / \sigma$. Thus we plotted the quantities $\varepsilon_{k} f /\left|p-p_{\mathrm{c}}\right|^{t}$ as a function of $f /\left|p-p_{\mathrm{c}}\right|^{t+s}$ $(f=\omega / 2 \pi)$. In 2D, we used the equality $t=s=1.35$ and in $3 \mathrm{D}$ we tried three values of $t=1.6$, 1.8 and 2. The results are displayed in figures 7-10 and we have some surprise. In $2 \mathrm{D}$, all the points correspondent to different values of $\left|p-p_{c}\right|$ are all on the same line for $\varepsilon_{1}$ as well for $\varepsilon_{2}$. It is a perfect verification of the scaling function (1). At low frequency the slope for $\varepsilon_{1}$ is 1 and for $\varepsilon_{2}$ is 2 , in accordance with (2). However, we do not reach the region for which $F$ is given by (3), since the slopes are always larger than $t /(t+s)=1 / 2$. Instead of a cross-over from (2) to (3), we have a very large intermediate region (five orders of magnitude in $f /\left|p-p_{c}\right|^{t+s}$ !).

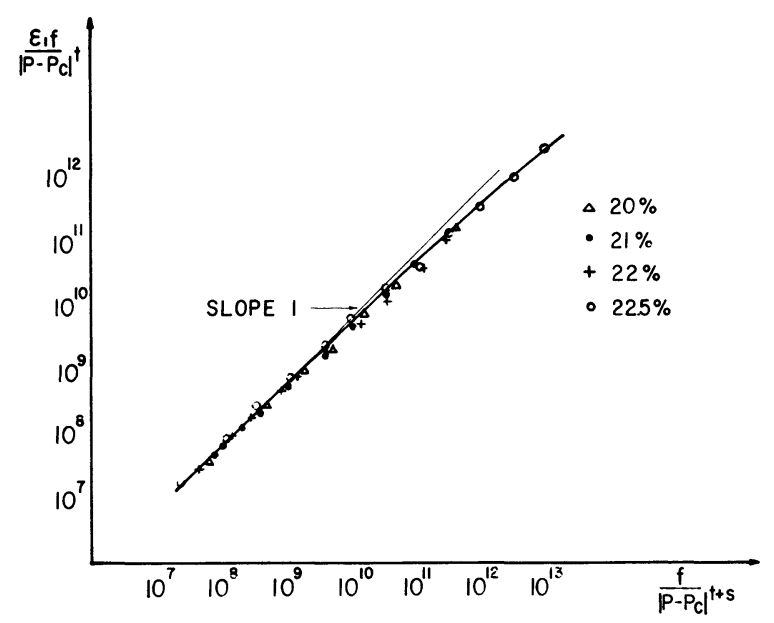

Fig. 7. - Scaling for $\varepsilon_{1}$ in $3 \mathrm{D}$.

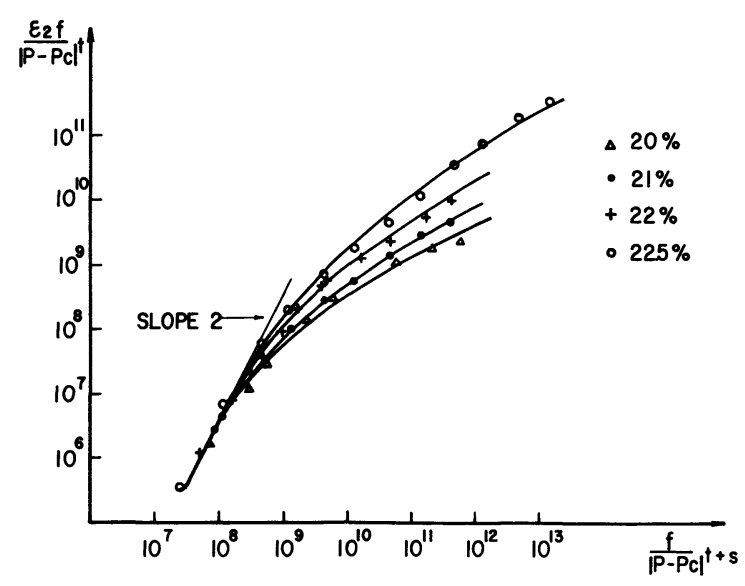

Fig. 8. - Scaling for $\varepsilon_{2}$ in 3D. 


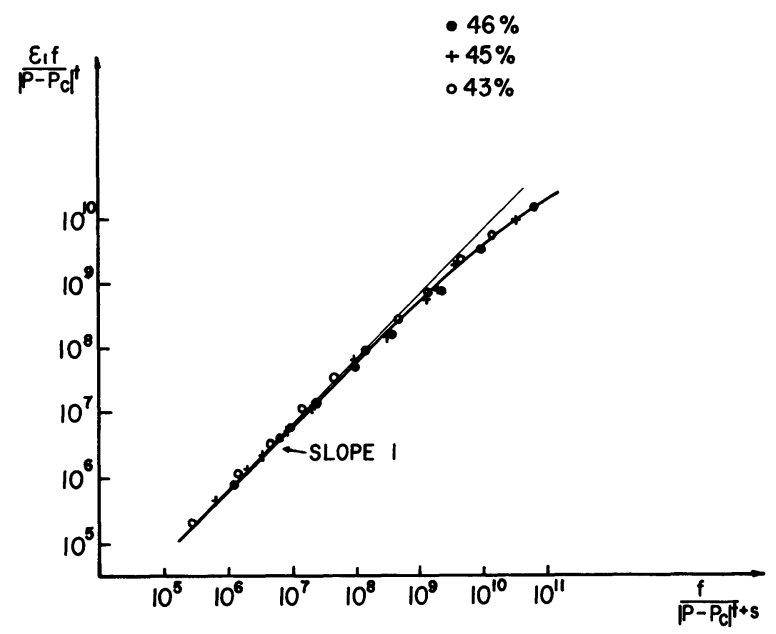

Fig. 9. - Scaling for $\varepsilon_{1}$ in 2D.

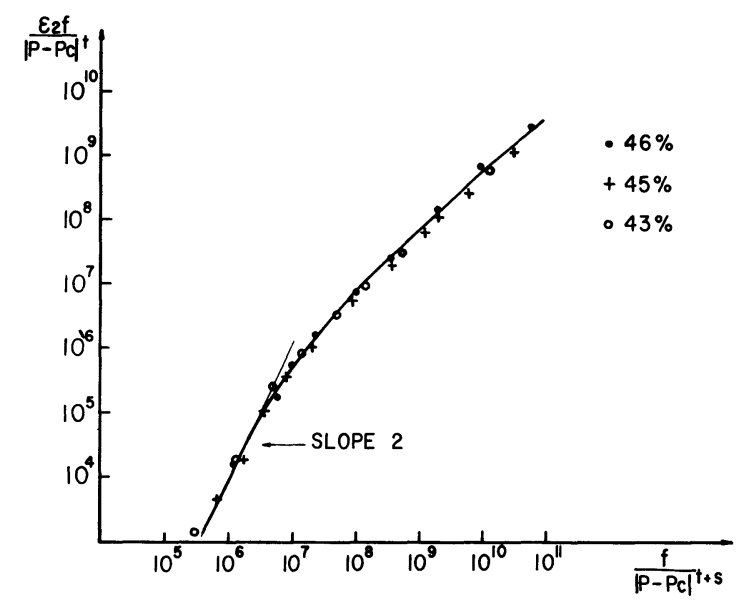

Fig. 10. - Scaling for $\varepsilon_{2}$ in $2 \mathrm{D}$.

It is possible to approximate the scaling functions $B_{k}$ by the following expression

$$
(k=1,2) \quad B_{k}(x) \sim x^{\alpha_{k}(x)}
$$

with $\alpha_{k}$ varying from 2 to $1 / 2$ for $\varepsilon_{2}$ and from 1 to $1 / 2$ for $\varepsilon_{1}$. But $\alpha_{1}=\alpha_{2}$ for $x=f /\left|p-p_{c}\right|^{t+s}$ $\gtrsim 10^{7}$. In these regions, $F$ is also given by $F(z) \sim z^{\alpha(z)}$. Considering that the maximum of $\varepsilon_{2}$ occurs when $\alpha_{2}\left(f /\left|p-p_{c}\right|^{t+s}\right)=1$, we see that our results are in agreement with $R(2 \mathrm{D})=t+s$.

In $3 \mathrm{D}$, we present the results for $t=1.8$, since this value gives the best result for $\varepsilon_{1}$. However, for $\varepsilon_{2}$ none of the values of $t$ gives the points to be aligned on the same line. Only at low frequency the points are aligned but for increasing the quantity $f /\left|p-p_{\mathrm{c}}\right|^{t+s}$, we have different lines for different $p-p_{c}$. We conclude : a) the scaling theory is verified only at low frequency 
(the slope for $\varepsilon_{1}$ is one and two for $\varepsilon_{2}$ ); b) at higher frequency there is a deviation from the scaling theory, which is manifested in $\varepsilon_{2}$. This explains why the exponent $R$ is different from $t+s$.

In conclusion, two remarks : 1) recently, Gefen et al. [7] suggested that the anomalous diffusion in percolative systems may be of importance for the understanding of the AC conduction. Clearly, in 2D there is no place for such a suggestion, since this gives different behaviour from that predicted by the scaling theory. But the discrepancy in 3D could be the result of neglecting the anomalous diffusion. 2) We did not reach the region for which (4) is valid. But it is not sure that it is possible to reach it. If $\omega_{p}>\omega_{\infty}$ the region where $\varepsilon_{1}$ is constant is reached first and (4) is never observed. This suggests that the best way of verifying scaling is to proceed as in this Letter and not considering the curves $\varepsilon_{1}(\omega)$ or $\varepsilon_{2}(\omega)$ at different values of $p$, as made by Laibowitz and Gefen [8].

\section{References}

[1] Dubrov, V. E., Levinshtein, M. E. and Shur, M., Sov. Phys. J.E.T.P. 43 (1976) 1050.

[2] Erros, E. L. and Shkцovski, B. I., Phys. Status Solidi (b) 76 (1976) 475.

Bergman, D. J. and Imry, Y., Phys. Rev. Lett. 39 (1977) 1222.

Webman, I., Jortner, J. and Cohen, M. H., Phys. Rev. B 16 (1977) 2593.

Stroud, D. and Bergman, D. J., Phys. Rev. B 25 (1982) 2067.

[3] Wilkinson, D., Langer, J. S. and Sen, P. N., Phys. Rev. B 28 (1983) 1081.

[4] Powell, M. J., Phys. Rev. B 20 (1979) 4194.

[5] Ottavi, H., Clerc, J., Giraud, G., Roussence, J., Guyon, E. and Mitescu, C. D., J. Phys. C 11 (1978) 1311.

[6] Benguigui, L., to be published.

[7] Gefen, Y., Aharony, A. and Alexander, A., Phys. Rev. Lett. 50 (1983) 77.

[8] Laibowitz, R. B. and Gefen, Y., Phys. Rev. Lett. 53 (1984) 380. 